\title{
Robotic Astronomy and the BOOTES Network of Robotic Telescopes
}

\author{
A. J. Castro-Tirado on behalf of the BOOTES Collaboration
}

\begin{abstract}
The Burst Observer and Optical Transient Exploring System (BOOTES), started in 1998 as a Spanish-Czech collaboration project, devoted to a study of optical emissions from gamma ray bursts (GRBs) that occur in the Universe. The first two BOOTES stations were located in Spain, and included medium size robotic telescopes with CCD cameras at the Cassegrain focus as well as all-sky cameras, with the two stations located $240 \mathrm{~km}$ apart. The first observing station (BOOTES-1) is located at ESAt (INTA-CEDEA) in Mazagón (Huelva) and the first light was obtained in July 1998. The second observing station (BOOTES-2) is located at La Mayora (CSIC) in Málaga and has been operating fully since July 2001. In 2009 BOOTES expanded abroad, with the third station (BOOTES-3) being installed in Blenheim (South Island, New Zealand) as result of a collaboration project with several institutions from the southern hemisphere. The fourth station (BOOTES-4) is on its way, to be deployed in 2011.
\end{abstract}

Keywords: Robotic Astronomy, Stellar Astrophysics, variable stars.

\section{Introduction}

Robotic astronomical observatories were first developed in the 1960s by astronomers after electromechanical interfaces to computers became common at observatories. Nowadays there more than 100 spread worldwide (Fig. 1). See [1] for an overview. Here are some important definitions in the field of Robotic Astronomical Observatories ${ }^{1}$ :

Automated scheduled telescope (Robot): A telescope that performs pre-programmed observations without immediate help of a remote ob- server (e.g. avoiding an astronomer moving the mount by hand).

Remotely operated (remote) telescope Robot: A telescope system that performs remote observations following the request of an observer.

Autonomous Robot (observatory): A telescope that performs various remote observations and is able to adapt itself to changes during task execution without any kind of human assistance (e.g. weather monitoring; the system must not endanger humans!).

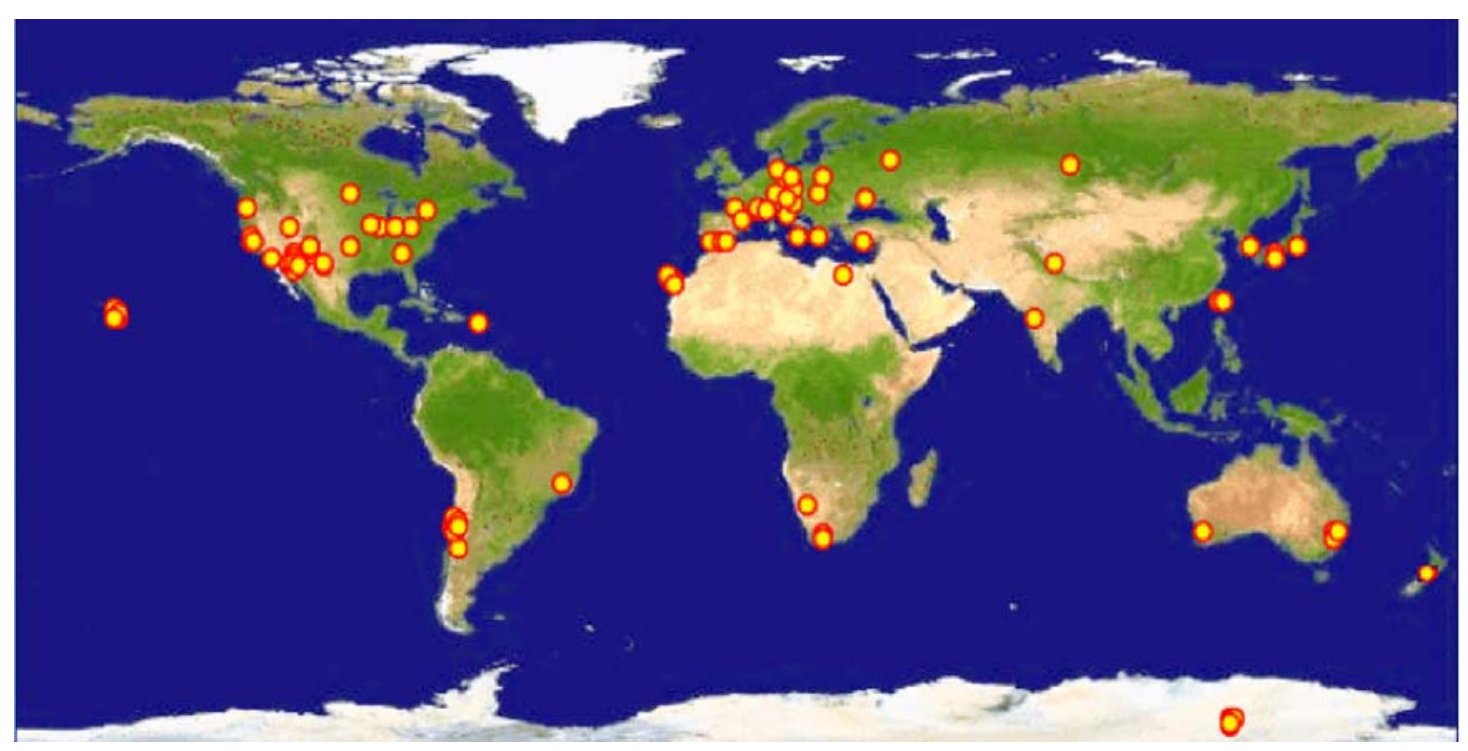

Fig. 1: Distribution of robotic telescopes in the world

\footnotetext{
${ }^{1}$ following the consensus reached after one hour of discussion amongst the 80 participants who attended the "Workshop in Robotic Autonomous Observatories", held in Málaga (Spain) on May 18-21, 2009.
} 
BOOTES (the Burst Observer and Optical Transient Exploring System, BOOTES), started in 1998 as a Spanish-Czech collaboration project [2] devoted to the study of optical emissions from gamma ray bursts (GRBs) that occur in the Universe. Nowadays it consists of 4 stations, three of them hosting $60 \mathrm{~cm}$ fast slewing robotic telescopes aimed at contributing significantly to various scientific fields.

\section{The BOOTES network of robotic telescopes}

\subsection{BOOTES-1}

The first robotic astronomical observatory in Spain was placed in the INTA's Estación de Sondeos Atmosféricos (ESAt) at the Centro de Experimentación de el Arenosillo in Mazagón (Moguer, Huelva). It has an extraordinary sky close to the Atlantic ocean with more than 300 clear nights a year, limited to the East by the Doñana National Park.

For the first two years after 1998, BOOTES provided rapid follow-up observations for more than 40 GRBs detected by Batse aboard the CGRO until it was turned off in May 2000. It consisted of a $0.2 \mathrm{~m}$ Schmidt-Cassegrain reflector telescope (at $\mathrm{f} / 10$ ) with a CCD camera at the Cassegrain focus, providing a $40^{\prime} \times 30^{\prime} \mathrm{FOV}$ and a couple of CCD cameras attached to the main optical tube providing a $16^{\circ} \times 11^{\circ} \mathrm{FOV}$.

Since 2001, with the new location of the existing enclosure $100 \mathrm{~m}$ away from the original site, and with the addition of the second enclosure (dubbed BOOTES-1B to distinguish it from BOOTES-1A, the old one), various setups have been accomplished, the current one, as of summer 2010, being as follows:

- A $0.3 \mathrm{~m}$ diameter Schmidt-Cassegrain reflector telescope (f/10) mounted on a Paramount mount with a narrow field CCD $(1528 \times 1024$ pix $)$ camera attached to the main optical tube: $30^{\prime} \times 20^{\prime}$ FOV.

- A wide field CCD camera (4096 $\times 4096$ pix $)$ attached to a $400 \mathrm{~mm} \mathrm{f} / 2.8$ lens providing a $5^{\circ} \times 5^{\circ}$ FOV.

- An all-sky CCD camera (CASANDRA-1): $180^{\circ}$ FOV. See [3].

\subsection{BOOTES-2}

The BOOTES-2 robotic astronomical station was officially opened on 7 Nov 2001 and it is located at CSIC's Estación Experimental de La Mayora in Algarrobo Costa (Málaga). It is limited to the South by the Mediterranean sea and to the North by the Tejeda-Almijara Mountains Nature Park with Maroma peak (2.068 m. a.s.l.).

Unlike the two domes of the BOOTES-1 station $200 \mathrm{~km}$ away, its dome is controlled by a hydraulic opening system controlled automatically according to the existing weather conditions.

BOOTES-2 at first hosted a 0.3-m SchmidtCassegrain reflector telescope (f/10), which was replaced in 2009 by a $0.6 \mathrm{~m}$ Ritchey-Chrétien fast slewing telescope. This was officially opened on 27 Nov 2009.

Thus, the new configuration in the BOOTES-2 station has the following instruments:

- The TELMA (TELescopio MAlaga) RitcheyChrétien reflector telescope $(0.6 \mathrm{~m}, \mathrm{f} / 8$, see Fig. 2) with an EMCCD narrow field camera with variou filters (clear, Johnson R., Sloan g'r'i' and UKIRT $\mathrm{Z}$ and $\mathrm{Y}$-band filters) providing a $10^{\prime} \times 10^{\prime} \mathrm{FOV}$.

- An all-sky camera (CASANDRA-2) providing a $180^{\circ} \mathrm{FOV}$.

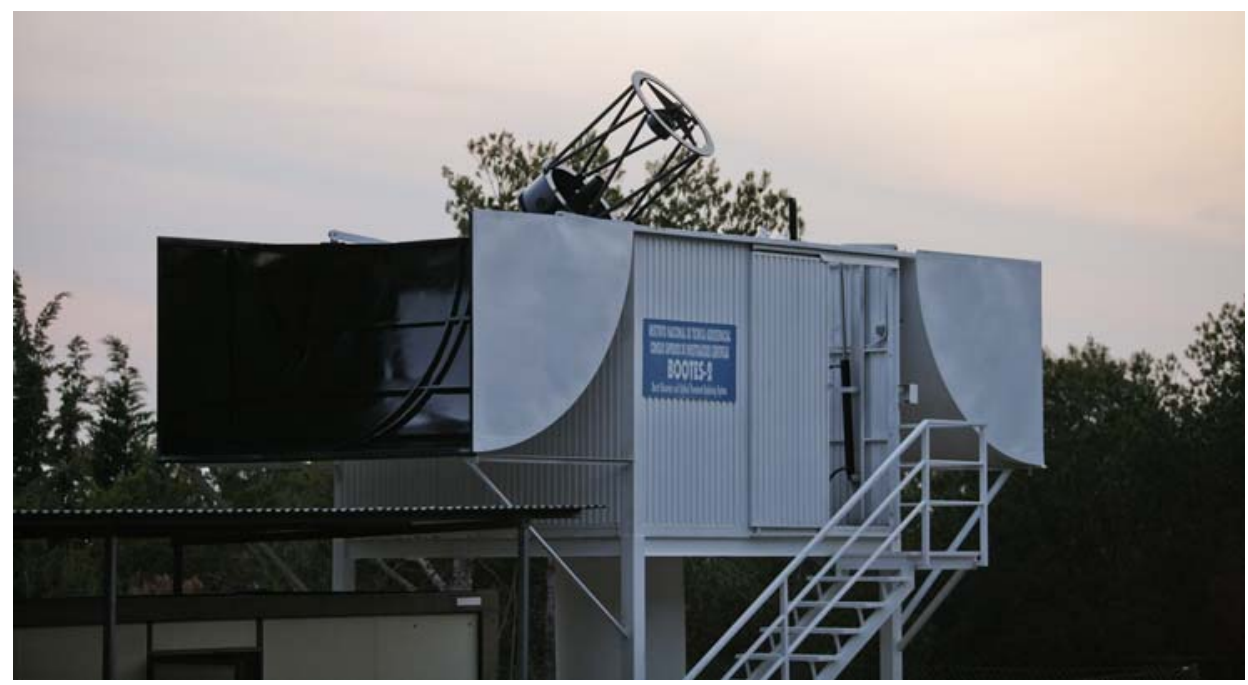

Fig. 2: The TELMA $0.6 \mathrm{~m}$ telescope at the BOOTES-2 astronomical station 


\subsection{BOOTES-3}

The BOOTES-3 robotic astronomical station was the first installation of the BOOTES Network outside Spain. It was officially opened on 27 Feb 2009. It is located at Vintage Lane, Blenheim (New Zealand), one of the best places for observing the night sky in the southern hemisphere.

Similarly to the BOOTES-1 station, its dome is controlled by electric motors, which are controlled automatically according to the existing weather conditions.

BOOTES-3 hosts a $0.6 \mathrm{~m}$ Ritchey-Chrétien fast slewing telescope, which is dubbed the YA (YockAllen) Telescope, in honour of Prof. Phil A. Yock and Eng. Bill H. Allen, in recognition of their encouraging support.

The BOOTES-3 station has the following instruments:

- The YA (Yock-Allen) Ritchey-Chrétien reflector telescope $(0.6 \mathrm{~m}, \mathrm{f} / 8)$ with an EMCCD narrow field camera (clear, Johnson B, Sloan g'r'i' and UKIRT $\mathrm{Z}$ and Y-band filters) providing a $10^{\prime} \times 10^{\prime} \mathrm{FOV}$

- The All-sky camera (CASANDRA-3) providing a $180^{\circ}$ FOV. See Fig. 3 .

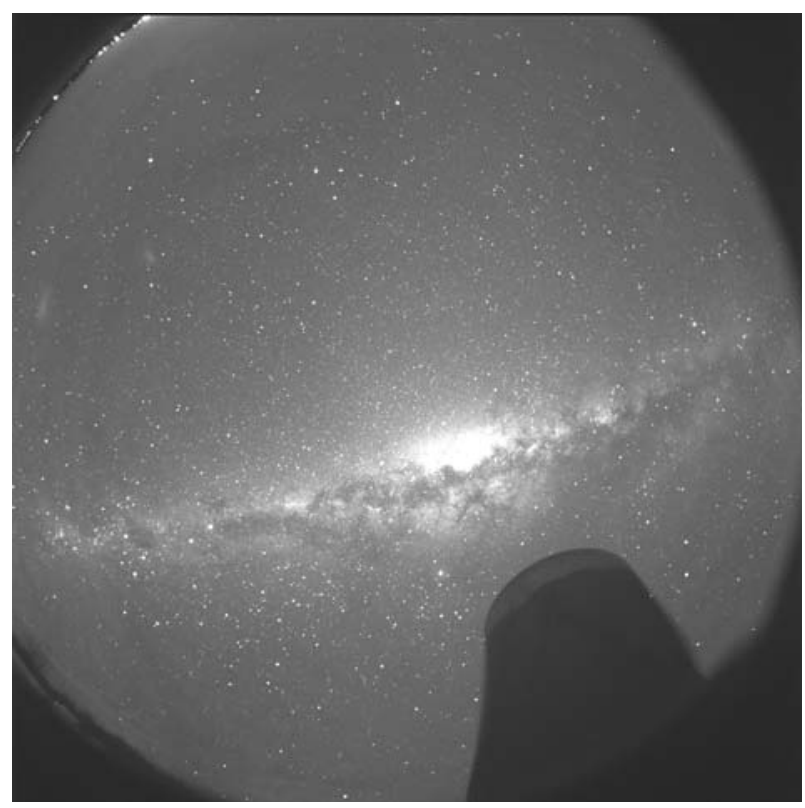

Fig. 3: The YA $0.6 \mathrm{~m}$ telescope in Blenheim (New Zealand) depicted against the centre of the Milky Way in an image recorded by CASANDRA-3. The fourth station (BOOTES-4) will be deployed in 2011

\section{BOOTES Scientific Goals}

The BOOTES scientific goals are multifold, and are detailed below.

\subsection{Observation of the GRB error box simultaneously to GRB occurrence}

Although the first detected optical counteparts were not brighter than 19th mag a few hours after the burst, there have been several GRBs for which the optical transient emission has been detected simultaneously to the gamma-ray event, with magnitudes in the range $5-10$. The faint transient emission that was detected a few hours after the event is a consequence of the expanding remnant that the GRB leaves behind it. This provides information about the surrounding medium, but not about the central engine itself. The fast slewing $0.6 \mathrm{~m}$ BOOTES telescopes are producing important results in this field [4]. See Fig. 4.

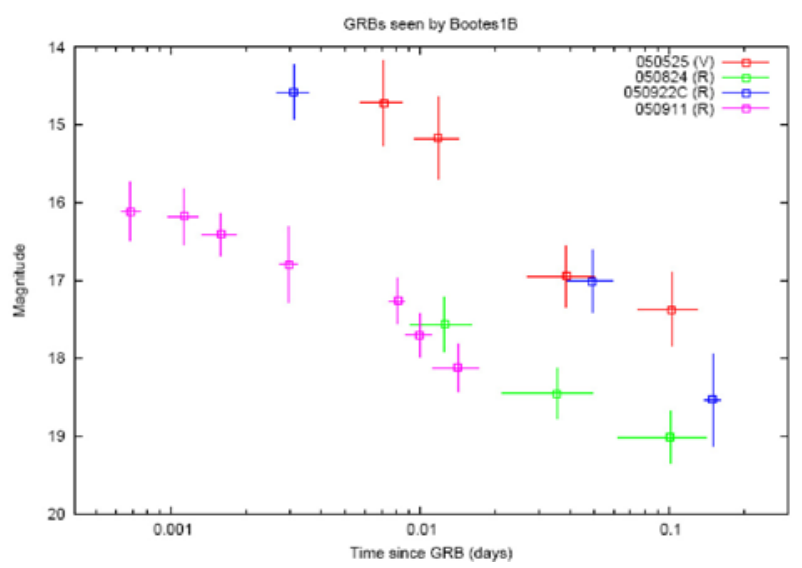

Fig. 4: Optical afterglow lightcurves of some GRBs detected by BOOTES and rapidly imaged (within $1 \mathrm{~min}$ ) after detection by scientific satellites

In this respect, coordinated observations of GRBs in various filters is most essential, as only a few GRBs have exceptionally bright optical counterparts. Observers are of course interested in collecting as much data as possible, with the best possible resolution.

One of the goals of the observers is to take spectra of the transient while it is bright enough, so that the transient redshift and other properties can be measured. Using data taken with different filters, one can construct the spectral energy distribution of the event and estimate the object redshift. Networked RTS telescopes (like BOOTES) at favourable locations can simultaneously observe objects in different filters.

The idea is to enable these telescopes to communicate with each other and provide simultaneous images in two or more filters. This system should balance the need to take some data with the possibility of taking data in multiple filters. This can be achieved by sending commands to take images in different filters when the system knows that it has at 
least some images of the event. This kind of decision is best made in a single component-observation coordinator.

The coordinator will be connected to two or more telescope nodes. It will collect information from GCN and from all connected nodes. A node will report to the coordinator when it receives a GCN notice, when it starts its observation and as soon as it gets an image passed through the astrometry and it contains the whole error area of the GRB. It will also report when the transient detection software identifies a possible optical transient.

When the coordinator receives messages about correct observation by two telescopes, it will decide which filter should be followed at which telescope, and will send out commands to carry out further observations. The coordinator will periodically revisit its observing policy, and send out commands to change filter accordingly.

As the system is "running against the clock" for the first few minutes after the GRB event, trying to capture the most interesting part of the transient light curve, it cannot wait for completion of the transient source analysis. In the case of two telescopes, the coordinator will command different filters as soon as it knows that both telescopes have acquired the relevant field. The current astrometry routines take a few seconds to run, and it is expected that observations with different filters can already have started within this time-frame.

\subsection{The detection of optical flashes (OTs) of cosmic origin}

These events could be unrelated to GRBs and could constitute a new type of different astrophysical phenomenon (perhaps associated to QSOs/AGNs). If some of them are related to GRBs, the most recent
GRB models predict that there should be a large number of bursting sources in which only transient X-ray/optical emission should be observed, but no gamma-ray emission. The latter would be confined in a jet-like structure and pointing towards us only in a few cases.

\subsection{Monitoring a range of astronomical objects}

These are astrophysical objects ranging from galactic sources such as comets (Fig. 5), cataclysmic variables, recurrent novae, compact X-ray binaries to extragalactic sources such as distant supernovae and bright active galactic nuclei. In the later case, there are hints that sudden and rapid flares occurs, though of smaller amplitude.

\section{Networking}

One step further from GRB observation is coordinated observation of targets - e.g. observation of variable stars for more than 12 hours (i.e. taking advantage of telescopes in different time zones). The observer should contact the coordinator, and either add a new target, or select a predefined target which he/she wants to observe. The coordinator should list for the observer telescopes which can observe the target of his/her choice, and propose filters and exposure times.

The observer can then decide which telescopes are to be used, and the coordinator will send observation requests to the nodes, and will collect back information about observation progress. Currently only observer-selected coordinated observations are envisioned. When that works properly, the observer can be replaced by network scheduling software.

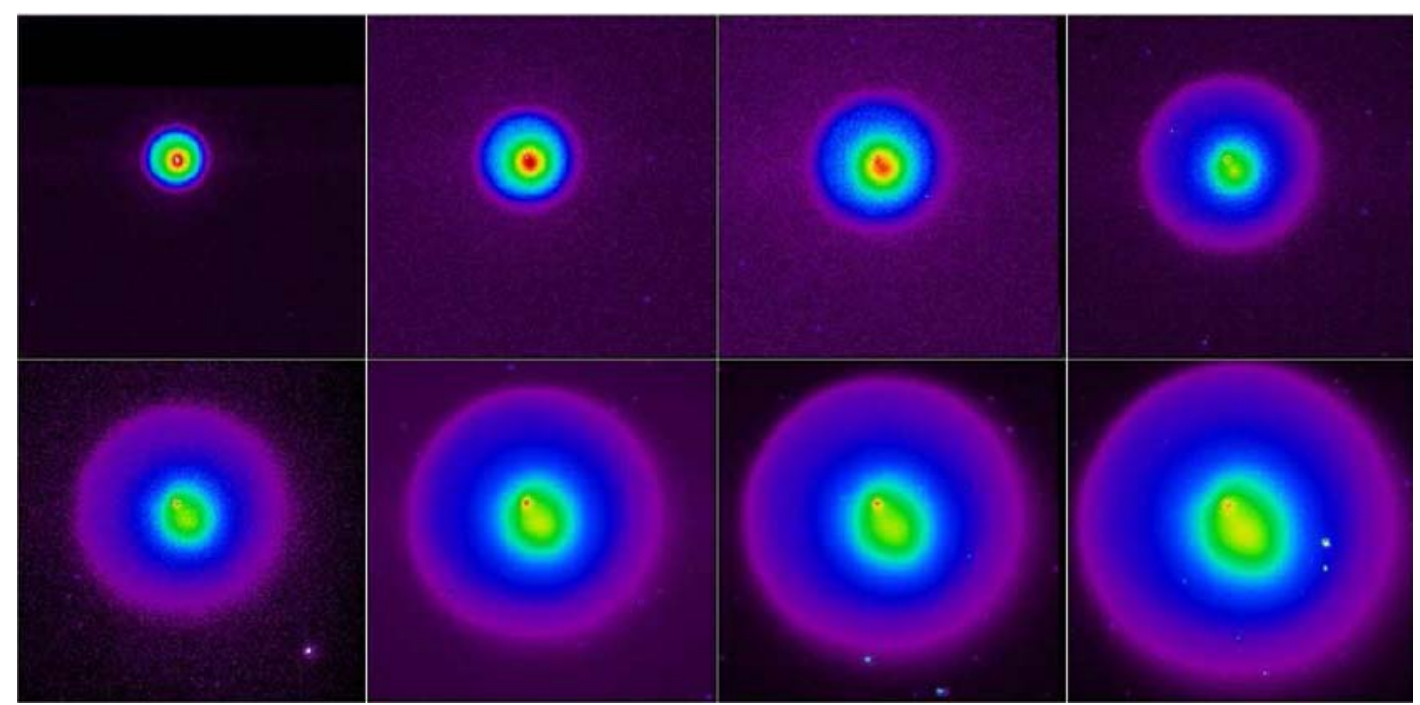

Fig. 5: The evolution of comet $17 \mathrm{P} /$ Holmes following the October 2007 outburst, imaged on a nightly basis with the BOO-2 telescope in Spain. The FOV is $10^{\prime} \times 10^{\prime}$ in all frames 


\section{Conclusions}

Robotic Telescopes are opening a new field in Astrophysics in terms of optimizing the observing time, with some of them able to provide pre-reduced data. The big advantage is that they can be placed in remote locations where human life conditions will be hostile (Antartica now, the Moon in the near future). BOOTES (http://www.iaa.es/bootes) is an example of such a telescope system. Technological development in various fields is much involved, and some robotic astronomical observatories are moving towards intelligent robotic astronomical observatories.

One immediate application of small/medium size robotic telescopes is in the study of GRBs, which can be considered the most energetic phenomenon in the Universe. In combination with space missions like Integral, Swift andFermi, they are used for triggering larger size instruments in order to perform more detailed studies of host galaxies and intervening material on the line of sight. These robotic astronomy observatories will provide a unique opportunity to unveil the high-z Universe in years to come.

\section{Acknowledgement}

We are very grateful for the support given by the Space Sciences and Electronics Technologies Department at INTA, through project IGE 4900506, to the special action DIF2001-4256-E supported by the Technology and Science Ministry (MCyT), and also to the Spanish research projects AYA2001-1708, AYA2002-0802, AYA2004-01515, AYA2007-6377 and AYA 2009-C14000-C03-01 granted by the Spanish Ministry of Science and Education and Innovation and Technology (with FEDER funding). The development of the BOOTES Network has been also possible thanks to the support of Junta de Andalucía through Excellence Research Projects P06FQM-2192 and P07-TIC-3094. The Czech contribution is supported by the Ministry of Education and Youth of the Czech Republic, Projects ES02 and ES36.

\section{References}

[1] Castro-Tirado, A. J.: Robotic Autonomous Observatories: A Historical Perspective, in $A d$ vances in Astronomy, issue on Robotic Astronomy, (edited by A.J. Castro-Tirado et al.), article id. 570489, 2010. (http://www.hindawi.com/ journals/aa/2010/570489.html)

[2] Castro-Tirado, A. J. et al.: The Burst Observer and Optical Transient Exploring System (BOOTES), A\&AS 138, 583, 1999.

[3] Castro-Tirado, A. J. et al.: A very sensitive all-sky CCD camera for continuous recording of the night sky, in Advanced Software and Control for Astronomy II. Edited by Bridger, Alan; Radziwill, Nicole M. Proceedings of the SPIE, Vol. 7 019, 2008, pp. 70191V-70191V-9.

[4] Jelínek, M. et al.: Four Years of Realtime GRB Follow-up by BOOTES-1B (2005-2008), in Advances in Astronomy, special issue on Robotic Astronomy (edited by A. J. Castro-Tirado et al.), 2010, arXiv1001.2147.

Alberto J. Castro-Tirado

E-mail: ajct@iaa.es

IAA-CSIC, Glorieta de la Astronomía s/n

E-18008 Granada, Spain 